\title{
Comparison of Transvaginal Ultrasound, Saline Infusion Sonohysterography versus Diagnostic Hysteroscopy in Evaluation of Endometrial Cavity Pathology amongst Women with Abnormal Uterine Bleeding in Low Resource Setting
} \author{
Rafique B. Parkar ${ }^{3}$, Elijah Kwasa ${ }^{4}$ \\ ${ }^{1}$ Department of Obstetrics \& Gynaecology, University of Nairobi, Nairobi, Kenya \\ ${ }^{2}$ Department of Anatomy, University of Nairobi, Nairobi, Kenya \\ ${ }^{3}$ Consultant Obstetrician \& Gynecologist, Nairobi, Kenya \\ ${ }^{4}$ Consultant Radiologist, Nairobi, Kenya \\ Email: jaynicd@gmail.com, joewanyoike@yahoo.co.uk
}

Jayni Dedhia ${ }^{1}$, Gichuhi J. Wanyoike ${ }^{1}$, Ojwang B. Shadrack, Madadi M. Obimbo ${ }^{2}$,

How to cite this paper: Dedhia, J., Wanyoike, G.J., Shadrack, O.B., Obimbo, M.M., Parkar, R.B. and Kwasa, E. (2020) Comparison of Transvaginal Ultrasound, Saline Infusion Sonohysterography versus Diagnostic Hysteroscopy in Evaluation of Endometrial Cavity Pathology amongst Women with Abnormal Uterine Bleeding in Low Resource Setting. Open Journal of Obstetrics and Gynecology, 10, 644-656. https://doi.org/10.4236/ojog.2020.1050058

Received: April 14, 2020

Accepted: May 5, 2020

Published: May 8, 2020

Copyright $\odot 2020$ by author(s) and Scientific Research Publishing Inc. This work is licensed under the Creative Commons Attribution International License (CC BY 4.0).

http://creativecommons.org/licenses/by/4.0/ (c) (i) Open Access

\section{Abstract}

Introduction: Hysteroscopy is currently the gold-standard protocol for evaluating patients with abnormal uterine bleeding (AUB). Unfortunately, though accurate, its adoption in low-resource countries such as in Africa is limited due to lack of equipment and qualified personnel. As such, there is a need for an alternative diagnostic procedure that is as accurate as hysteroscopy, but also affordable, easy to administer, and acceptable by women with endometrial pathologies. Transvaginal Sonography (TVS) and Saline Infusion Sonohysterography (SIS) are proposed. However, their diagnostic accuracy versus hysteroscopy has not been determined in low resource setting. Objective: To compare the diagnostic efficacy of TVS and SIS versus diagnostic hysteroscopy in evaluation of endometrial pathology among pre-menopausal and post-menopausal women and to determine the etiology of AUB amongst these women. Methodology: A prospective cohort study was done at a hospital in Nairobi, Kenya between May and September 2019. Forty patients with AUB were recruited using consecutive sampling, and women who consented were recruited. The etiology of AUB was recorded. All participants underwent TVS, SIS and Diagnostic Hysteroscopy (DH) evaluation in the first half of the menstrual cycle and the findings recorded on a patient's information 
sheet. The sociodemographic and bleeding characteristics of patients and the outcomes of TVS, SIS, and DH evaluations were also recorded and the data was analysed using version 5 of the Software for Statistics and Data Science (STATA). Summary statistics on the etiology of AUB were presented and the sensitivity of TVS and SIS versus DH as the gold-standard evaluated using two by two tables and the ROC curve. Results: The mean age of participants was $38.1 \pm 8.8$ years, range of $25-71$ years. Heavy Menstrual Bleeding (HMB) was reported in $70.0 \%$ of participants, while about $12.0 \%, 7.5 \%$, and $7.5 \%$ had post-menopausal bleeding, amenorrhea, and hypomenorrhea. The incidence of submucosal fibroids and endometrial polyps was $17.5 \%$ and $15.0 \%$ via TVS, $47.5 \%$ and $20.0 \%$ via SIS and $52.5 \%$ and $20 \%$ via DH respectively. The overall sensitivity, specificity, Positive Predictive Value (PPV), Positive Predictive Value (NPV), and diagnostic accuracy of SIS were 92.1\%, 83.3\%, 96.9\%, $62.5 \%$, and $90.0 \%$ while TVS was $38.2 \%, 100 \%, 100 \%, 22.2 \%$, and $47.5 \%$. The sensitivity, specificity, PPV, and NPV of TVS in diagnosis of endometrial polyp were $75.0 \%, 100 \%, 100 \%$, and $94.0 \%$. SIS did better with a sensitivity, specificity, PPV, and NPV of $100 \%, 100 \%, 100 \%$, and $100 \%$. Conclusion: Our data suggests SIS had a higher diagnostic accuracy than TVS and showcased a comparable diagnostic accuracy to hysteroscopy. SIS is more suitable safe alternative technique for investigating AUB in pre/post-menopausal women in low resource setting where hysteroscopy is unavailable or unaffordable.

\section{Keywords}

Abnormal Uterine Bleeding, Transvaginal Sonography, Saline Infusion Sonohysterography, Hysteroscopy

\section{Background}

Abnormal Uterine Bleeding is among the most common indications for gynecological surgical intervention in both premenopausal and postmenopausal women [1], with studies reporting that $70 \%$ of gynecological consultations of premenopausal women are for AUB [2]. Several tools and approaches have been developed to ensure a proper diagnosis of endometrial cavity anomalies, encompassing hysteroscopy, SIS, and TVS [3] [4] [5] [6].

Hysteroscopy is a diagnostic and therapeutic intervention for the detection and treatment of the causal factors for AUB [7]. Even though hysteroscopy is regarded as the gold standard for defining the causes of AUB because it allows direct visualization of the endometrial cavity and facilitating excision of a small portion of a suspected abnormality, it is an invasive expensive procedure [8]. Moreover, it does not offer additional information on adnexa and myometrium [9], and has been associated with severe pain and discomfort during its administration and possibility of complications, which lengthens hospital stay, increases acquisition of nosocomial infections, and increases the cost of management of co-morbidities that are associated pain [10]. During its administration, it can induce a plethora of complications such as thrombosis, infection, bowel or 
bladder damage, and hemorrhage [11]. Furthermore, hysteroscopy is not readily available in resource limited countries and is also technically challenging.

Transvaginal Ultrasound is sensitive in the detection of endometrial carcinoma and hyperplasia [12] [13], submucosal fibroids [14], and endometrial polyps [8]. The procedure also facilitates clear observation of the endometrial cavity, but has a high false positive and false negatives rate [3] [15] and does not isolate focal uterine lesions well [16] [17]. TVS is a suitable alternative to hysteroscopy due to its safety and ease of use, but has been found to have a high rate of false negatives in detection of focal intrauterine abnormalities.

Saline infusion sonohysterography (SIS) is a simple and precise procedure that entails gradual administration of sterile saline into the uterine cavity through a catheter inserted via the cervix [5]. The procedure generates better ultrasound scans of the endometrial cavity than traditional approaches like TVS with Dekroon et al. [18] reporting a success rate of $93 \%$. SIS has been cited to be less painful and thus more acceptable than TVS and DH [1] [16] [17]. SIS also offers detailed images of endometrial cavities than TVS, is cheaper, and can distinguish focal endometrial lesions that need a directed biopsy with a lower risk of complications and or prolonged hospital admissions, which makes it a suitable substitute of $\mathrm{DH}$ as an initial diagnostic procedure for women suffering from AUB. Whereas SIS has been touted as the best alternative to hysteroscopy, its efficacy in diagnosing some pathologies has been questioned [9] [19]. Hence, there is a need to investigate SIS efficacy conclusively.

Endometrial pathologies are associated with high hospitalization rates, skyrocketing healthcare cost, high morbidity, and mortality rates. This calls for a study to find the most appropriate tool in diagnosis of endometrial pathology in terms of cost effectiveness, accuracy in diagnosis and complications in low resource setting.

The focus of the study is to evaluate the diagnostic efficacy of TVS and SIS versus DH for the evaluation of endometrial cavity pathologies among premenopausal and postmenopausal women in a low resource setting.

\section{Objective}

To compare the diagnostic efficacy of TVS and SIS versus diagnostic hysteroscopy in the evaluation of endometrial pathology among pre-menopausal and post-menopausal women in low resource setting.

\section{Methods}

A prospective, cohort study for assessing the demographic pattern of undiagnosed Abnormal Uterine Bleeding and the efficacies of TVS, SIS in diagnosing abnormalities of uterine cavity as compared to hysteroscopy. The study was carried out in a Minimal Access Day Care hospital in Nairobi, Kenya. The study population was premenopausal and postmenopausal patients aged 19 to $75 \mathrm{yrs}$ who presented with a diagnosis of AUB from May 1st, 2019 and September 30th, 2019. The sample size of the study population was 40 and was calculated using Buderer's formula for sample size calculation. The study was reviewed and ap- 
proved by the Kenyan ethical authority (Kenyatta National Hospital/University of Nairobi Ethics Research Committee) with respect to scientific content and compliance with applicable research and human subjects' regulations. Patients who consented for the study were registered and informed about the procedures of DH, TVS and SIS by the research assistants or principal investigator. Data collected included the age, BMI, parity, medical surgical obstetric \& gynecological history and the findings of TVS, SIS and hysteroscopy. Information collected was handled with Belmont's principles of confidentiality. Each participant was allocated a unique study identification coded number for confidentiality.

The optimal timing for TVS and SIS depended on the clinical presentation. In a woman who had regular menstrual cycles, TVS and SIS was typically done early in the follicular phase of the cycle, after cessation of menstrual flow, but no later than day 10 of the cycle this is because a thin endometrium gave proper visualization of focal lesions. The secretory phase was avoided because folds of the endometrium can mimic small fibroids or polyps or focal areas of endometrial hyperplasia. In cases where there is prolonged or irregular bleeding the procedure was carried out when there was no active bleeding.

TVS: Patient was asked empty the bladder and lie on an examination table in lithotomy position for transvaginal ultrasound. A 3D-TVS was conducted with Seimens Acuson NX3 USG machine using a transvaginal probe of $5.0-8.0 \mathrm{MHz}$ which was covered with a plastic or latex sheath and lubricated. The tip of the transducer was inserted into the posterior fornix. The transducer was gently turned and angled to measure the endometrial cavity allowing clear visualization in longitudinal and transverse planes by TVS the endometrial lining. The endometrium was found to be abnormal, if $>5 \mathrm{~mm}$ in postmenopausal women or $>12$ $\mathrm{mm}$ in a premenopausal patient [9]. Patients were subsequently subjected to SIS.

SIS: A SIS catheter (Sion-Test sonosalpingography device) was inserted into the uterine cavity following direct visualization, without dilating the cervix or use of local anesthesia. A vaginal probe was re-inserted in the posterior fornix of the vagina behind the catheter. Approximately $20-30 \mathrm{ml}$ of $0.9 \%$ saline was injected into the catheter to inflate the endometrial cavity allowing clear visualization in longitudinal and transverse planes by TVS. Upon completion of both TVS and SIS procedures the data was collected by the research assistants and filled in on the data collection form.

Diagnostic Hysteroscopy: The hysteroscopy was performed using Olympus A4673A 12-degree rigid hysteroscope under aseptic techniques and following all infection prevention and control guidelines. A rigid hysteroscope was introduced into the cervix under direct visualization followed by inflation with isotonic solution. Upon completion of both procedures data was collected by the research assistants and filled in on the data collection form to complete the form.

\section{Data Collection}

The data which collected from the eligible patients was entered and signed in the logbook by the investigators. Quality assurance was enhanced continuously throughout the study period to maximize on the validity and reliability of the 
findings. The questionnaires were checked and validity for completeness at the end of each day by the principal investigator during data collection period to ensure completeness and accuracy of data collected. The collected data was entered and analyzed using the STATA software version 23. Specificity, sensitivity, positive predictive values (PPVs), negative predictive values (NPVs), and diagnostic accuracy (DA) were computed and used to determine the diagnostic precision of TVS, SIS and hysteroscopy in the detection of endometrial pathologies. Further analysis was done and presented in form of receiver operating curves (ROC). A $p$ value of $<0.05$ was taken as statically significant.

\section{Results}

Out of 65 patients who presented with abnormal uterine bleeding, 12 were excluded due to incomplete records, ten patients declined consent, and three patients did not fit the inclusion criteria. Therefore 40 pre-menopausal and post-menopausal patients were recruited. The mean age of participants was 38.1 years \pm 8.8 years, range 25 - 71 years. Thirty-five patients (87.5\%) were of a pre-menopausal age and mainly of African origin (75\%).

Bleeding patterns distribution amongst the Study Participants with ABU is shown in Figure 1.

Twenty-eight 28 (70\%) of the patients presented with a history of heavy menstrual bleeding, 5 (12\%) presented with post-menopausal bleeding and $3(7.5 \%)$ presented with a history of hypomenorrhea and amenorrhea respectively.

The pathological findings of women with AUB are summarized in Table 1 below. Out of the $403 \mathrm{D}$-transvaginal ultrasounds that were conducted prior to SIS, 27 (67.5\%) showed Normal Cavity, 7 (17.5\%) submucosal fibroids, and 6 (15\%) endometrial polyps. TVS did not detect endometrial lesions such as endometrioid cyst (adenomyosis), cervical stenosis, Mullerian duct anomalies and synechiae. SIS evaluation showed a normal cavity in $8(20 \%)$ of participants, while $29(72.5 \%)$ cases had a submucous myoma (47.5\%), endometrial polyp (20.0\%), uterine adhesions (7.5\%), adenomyosis (endometroid cyst) (5\%) and cervical polyps (2.5\%). Two cases were diagnosed falsely with normal uterine cavity by SIS, but were diagnosed with abnormal uterine cavity on diagnostic hysteroscopy. However, hysteroscopic evaluation of the Uterine cavity showed no false negative cases as $6(15 \%)$ cases were diagnosed with normal cavity and uterine cavity abnormalities were diagnosed with synechiae, 5\% adenomyosis (endometroid cyst), $2.5 \%$ cervical polyp, 2.5 with Mullerian duct anomaly.

The overall sensitivity, specificity, PPV, NPV, and accuracy in diagnosing endometrial pathologies were $38.2 \%, 100 \%, 100 \%, 22.2 \%$, and $47.5 \%$. TVS demonstrated a low sensitivity in diagnosing submucosal fibroids (33.3\%) with a specificity, PPV, and NPV of $100 \%, 100 \%$, and $57.6 \%$ but a high sensitivity and specificity in diagnosing endometrial polyps (75\% and $100 \%$ respectively). The sensitivity, specificity, PPV, NPV, and accuracy for a normal endometrial pathology were $38.2 \% .100 \%, 100 \%$, and $100 \%$ (Table $2 \&$ Table 3 ). 


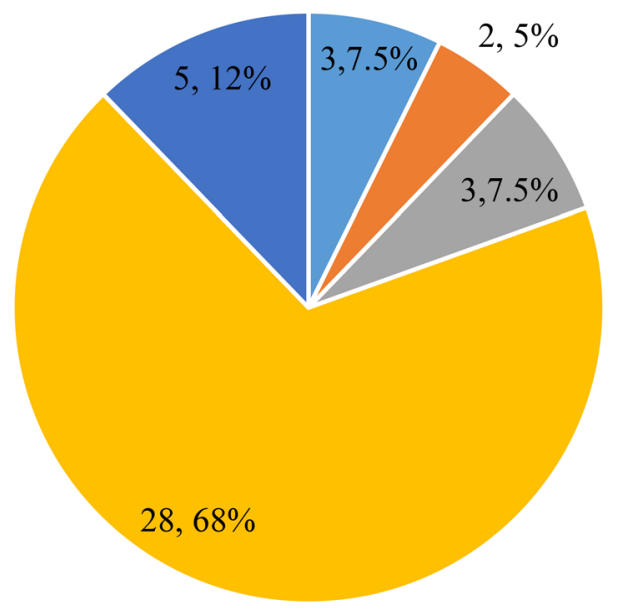

- Amenorrhea

- Hypomenorrhoea

Post-menopausal bleeding

Figure 1. Bleeding patterns of the study participants with AUB.

Table 1. TVS, SIS, and diagnostic hysteroscopy findings of premenopausal and postmenopausal women.

\begin{tabular}{cccc}
\hline & \multicolumn{3}{c}{ Frequency. $\boldsymbol{n}(\%)$} \\
\hline Normal & TVS & SIS & Diag. Hysteroscopy \\
\hline Sub mucosal fibroid & $27(67.5)$ & $8(20.0)$ & $6(15.0)$ \\
Endometrial polyp & $7(17.5)$ & $19(47.5)$ & $21(52.5)$ \\
Cervical polyp & $6(15)$ & $8(20.0)$ & $8(20.0)$ \\
Endometrioid cyst & $0(0.0)$ & $1(2.5)$ & $1(2.5)$ \\
Synechiae & $0(0.0)$ & $2(5.0)$ & $2(5.0)$ \\
Cervical stenosis & $0(0.0)$ & $3(7.5)$ & $6(15.0)$ \\
Mullerian duct anomalies & $0(0.0)$ & $3(7.5)$ & $3(7.5)$ \\
& $0(0.0)$ & $0(0.0)$ & $1(2.5)$
\end{tabular}

Table 2. Sensitivity and specificity of TVS versus hysteroscopy in evaluation of endometrial pathology in pre- and postmenopausal women.

\begin{tabular}{cccccc}
\hline Pathology & Sensitivity & Specificity & $\boldsymbol{P P V}$ & $\boldsymbol{N P V}$ & $\boldsymbol{D A}$ \\
\hline Overall & 38.2 & 100 & 100 & 22.2 & 47.5 \\
Normal & 38.2 & 100 & 100 & 22.2 & 47.5 \\
Submucosal Fibroid & 33.3 & 100 & 100 & 57.6 & 65.0 \\
Endometrial polyp & 75.0 & 100 & 100 & 94.1 & 95.0 \\
Synechia & 0 & 100 & - & 85.0 & 85.0 \\
Endometrial cyst & 0 & 100 & - & 95.0 & 95.0 \\
\hline
\end{tabular}

TVS: Transvaginal Ultrasound; PPV: Positive Predictive Value; NPV: Negative Predictive Value; DA: diagnostic accuracy. 
Saline infusion sonohysterography demonstrated high sensitivity (91.2\%), specificity (83.3\%), PPV (96.9\%), NPV (62.5\%), and accuracy (90.0\%) in diagnosing endometrial pathologies. In detecting endometrial polyps, the sensitivity, specificity, PPV, NPV and accuracy of SIS was $100 \%, 100 \%, 100 \%, 100 \%$, and $100 \%$. In detecting submucous myomas, its sensitivity, specificity, PPV and NPV were $90.5 \%, 100 \%, 100 \%, 90.5 \%$ respectively (Table 4, Figure 2).

Cervical polyps and cervical stenosis were incidental findings of SIS evaluation.

Overall, the sensitivity, NPV, and accuracy of SIS $(92.1 \%, 62.5 \%$, and $90.0 \%)$ were higher than TVS $(38.2 \%, 22.2 \%$, and $47.5 \%)$. However, TVS demonstrated a higher specificity (100\%) and PPV (100\%) than SIS (83.3\% and 96.9\%).

SIS demonstrated a higher sensitivity in detecting normal endocrine pathology (94.1\%), sub mucosal fibroids (90.5\%) and endometrial polyps (100\%) than TVS (38.2\%, 33.3\%, and $75.0 \%$ respectively). Even though the specificity of TVS in detecting the three pathologies (100\%) was comparable to SIS (100\%), SIS had a higher accuracy (Table 5).

Receiver Operating for the Overall sensitivity of using SIS in diagnosis of AUB taking hysteroscopy as the gold standard (Area under curve: 0.938 , Std error 0.038 , p value $<0.001$, C I $0.864-1.00$, kappa 0.737 , $\mathrm{p}<0.001)$.

Complications

There were no documented complications during Transvaginal scan, during or after Saline Infusion Sonohysterography whereas diagnostic hysteroscopy had 2 complications uterine perforation (fundal) and false passage created into the posterior wall.

Table 3. Sensitivity, specificity PPV and NPV of SIS versus hysteroscopy.

\begin{tabular}{cccccc}
\hline Pathology & Sensitivity & Specificity & $\boldsymbol{P P V}$ & $\boldsymbol{N P V}$ & $\boldsymbol{D A}$ \\
\hline Overall & 92.1 & 83.3 & 96.9 & 62.5 & 90.0 \\
Normal & 94.1 & 100 & 100 & 75.0 & 95.0 \\
Sub mucosal fibroids & 90.5 & 100 & 100 & 90.5 & 95.0 \\
Endometrial polyp & 100 & 100 & 100 & 100 & 100 \\
Endometrioid cyst & 100 & 100 & 100 & 100 & 100 \\
Synechiae & 50 & 100 & 100 & 91.9 & 92.5 \\
\hline
\end{tabular}

SIS: Saline infusion Sonohysterography; HS: Hysteroscopy; PPV: Positive Predictive Value; NPV: Negative Predictive Value; DA: diagnostic accuracy.

Table 4. Overall sensitivity, specificity, PPV, NPV, and accuracy of TVS versus SIS.

\begin{tabular}{cccccc}
\hline Pathology & Sensitivity & Specificity & PPV & NPV & Accuracy \\
\hline TVS & 38.2 & 100 & 100 & 22.2 & 47.5 \\
SIS & 92.1 & 83.3 & 96.9 & 62.5 & 90.0
\end{tabular}

SIS: Saline infusion Sonohysterography; TVS: Transvaginal ultrasound; PPV: Positive Predictive Value; NPV: Negative Predictive Value; DA: diagnostic accuracy. 
Table 5. Comparison of the sensitivity and specificity of SIS and TVS in detecting individual endometrial pathologies.

\begin{tabular}{ccccccccccc}
\hline & \multicolumn{1}{c}{$S I S$} & \multicolumn{1}{c}{$T V S$} \\
\hline Pathology & $S e$. & $S p$. & $P P V$ & $N P V$ & $D A$ & $S e$ & $S p$. & $P P V$ & $N P V$ & $D A$ \\
\hline Normal & 94.1 & 100 & 100 & 75.0 & 95.0 & 38.2 & 100 & 100 & 22.2 & 47.5 \\
Sub mucosal fibroids & 90.5 & 100 & 100 & 90.5 & 95.0 & 33.3 & 100 & 100 & 57.6 & 65.0 \\
Endometrial polyp & 100 & 100 & 100 & 100 & 100 & 75.0 & 100 & 100 & 94.1 & 95.0 \\
Endometrioid cyst & 100 & 100 & 100 & 100 & 100 & 0 & 100 & - & 85.0 & 85.0 \\
Synechiae & 50 & 100 & 100 & 91.9 & 92.5 & 0 & 100 & - & 95.0 & 95.0 \\
\hline
\end{tabular}

SIS: Saline infusion Sonohysterography; TVS: Transvaginal ultrasound; PPV: Positive Predictive Value; NPV: Negative Predictive Value; DA: diagnostic accuracy.

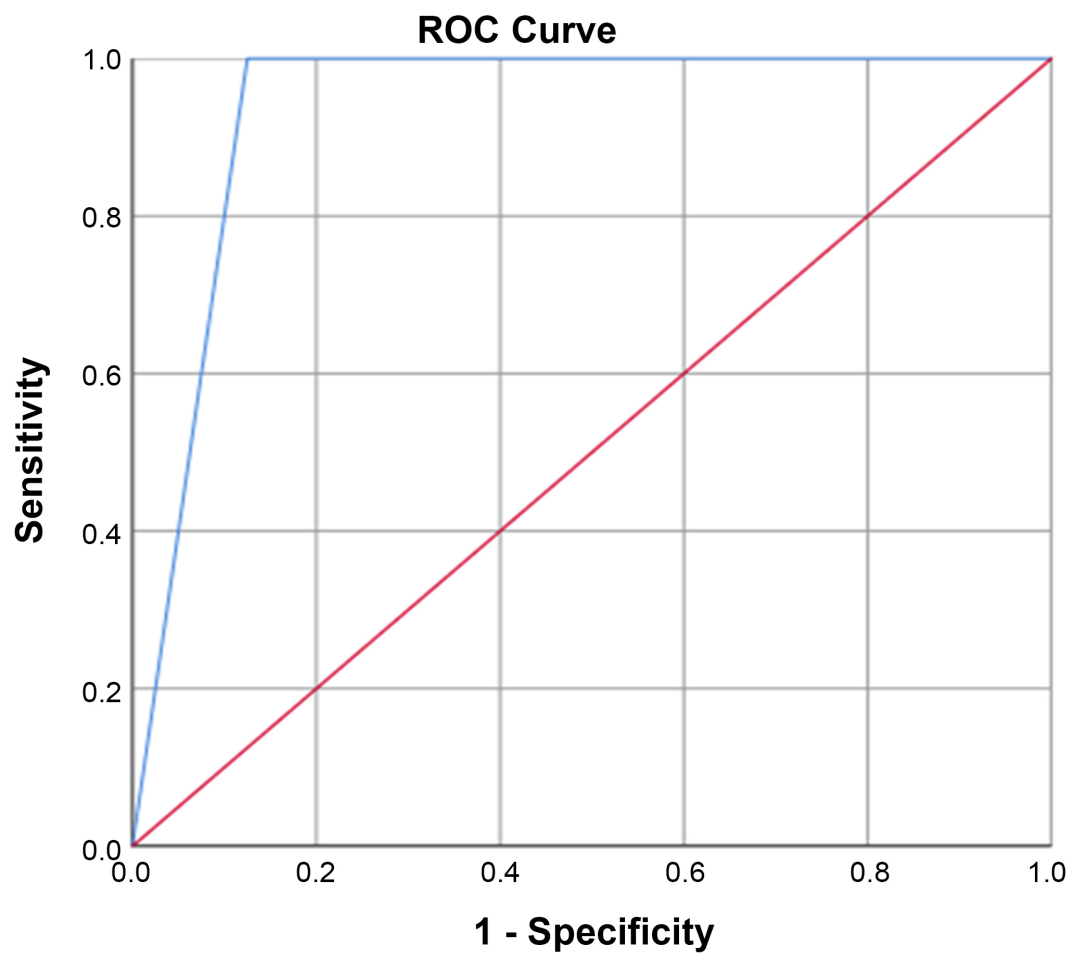

Diagonal segments are produced by ties.

Figure 2. SIS in diagnosis of AUB taking hysteroscopy as the gold standard.

\section{Discussion}

The management of abnormal uterine bleeding, a common symptom in women of all ages continues to pause a significant financial burden on healthcare resources especially in resource limited countries. Hence, efficient cost effective and technically easy method of diagnosing this condition is imperative; this is the focus of this study.

The sociodemographic characteristics of abnormal uterine bleeding have shown to have a variable pattern in different studies. The mean age of the study population presenting with AUB was $38.1 \pm 8.8$ (25 - 71), out of which $87.5 \%$ 
were premenopausal and most were of African descent (75\%).

Out of 40 patients with abnormal uterine bleeding, $70 \%$ had heavy menstrual bleeding (HMB), 12\% had postmenopausal bleeding, 7.5\% had amenorrhea and hypomenorrhea respectively, $5 \%$ had intermenstrual bleeding which was similar in studies conducted by Khan et al. in Saudi Arabia [1] and Rani et al. in India [20], in which HMB was the commonest presenting complaint at $73 \%$.

The 3D-TVS was able to detect endometrial lesions in only $32.5 \%$ of patients with undiagnosed abnormal uterine bleeding to be suffering from uterine pathologies. The pathologies that were noted on TVS were submucosal fibroid and endometrial polyps and other focal lesions such as endometrioid cyst, synechiae and cervical stenosis were not diagnosed. Though 2D/3D-TVS is the first imaging modality of choice for the evaluation of endometrial cavity in AUB of less than 12 weeks size uterus, it has limitations in detecting small lesions, location of myoma and in differentiating diffuse and focal lesion [20]. The sensitivity and specificity of TVS, SIS in detecting intracavitary abnormalities were 31.3 and $100 \%, 94.1 \%$ and $100 \%$; Dijkhuizen [21] reported a comparable diagnostic accuracy.

3D-Saline infusion sonohysterography was able to detect $80 \%$ of patients with undiagnosed abnormal uterine bleeding to be suffering from uterine pathologies. Most prevalent pathologies found in both pre- and postmenopausal age group was submucosal fibroids (47.5\%) and endometrial polyps (20\%) of the patient presenting with AUB. These findings were comparable to a study done in 2017 by Shaikh [22] where she documented $78 \%$ of patients who presented with undiagnosed AUB were found to have a uterine pathology with equal prevalence of polyps and submucosal fibroids.

Saline infusion sonohysterography in this study, the detection of endometrial pathology had sensitivity of $90.5 \%, 100 \%$ and $92.5 \%$ in diagnosis submucosal fibroids, endometrial polyps and endometrial lesions such as synechia, endometroid cyst was respectively. Its specificity in detecting the same pathology was $100 \%, 100 \%, 100 \%$ respectively. The positive and negative predictive values was 100:90.5, 100:100, and 100:85.7, demonstrating a higher sensitivity compared to the overall across women for all ages. The findings demonstrate a high sensitivity and specificity in diagnosing endometrial polyps and endometrioid cyst (100\%) but slightly lower sensitivity and specificity in diagnosing sub mucosal fibroids. These findings were comparable to other studies [19] [20].

Other pathologies diagnosed with SIS were endometroid cysts, synechiae, and cervical stenosis. Cervical stenosis was a diagnosis of exclusion made when there were 3 failed SIS procedures. These 3 patients underwent a Diagnostic hysteroscopy where the diagnosis of cervical stenosis and synechia was confirmed. This was also documented in a study done by Faryal Khan et al. in 2010 [1] in which he had 6 patients out of 101 patients with cervical stenosis, amongst these 1 was documented to have endometrial cancer.

Additionally, this study demonstrated a high area under curve on the ROC of $0.938, \mathrm{p}<0.001$ and the relatively high kappa coefficient of 0.737 further con- 
firming accuracy of using SIS in the identification of uterine lesions in comparison to using diagnostic hysteroscopy. These findings were seen in a systemic review and meta-analysis which demonstrated a high area under ROC curve of 0.97 in detection of intrauterine pathology [23]. Hence, diagnostic hysteroscopy should only a complimentary procedure in case of abnormal findings detected by other methods such as SIS and TVS.

Out of the 40 hysteroscopies that were conducted, $15 \%$ showed a normal cavity, $52.5 \%$ showed submucosal fibroids, $20 \%$ showed endometrial polyps, $5 \%$ showed andometrioid cyst, 5\% showed synechia and 2.5\% had Mullerian duct anomaly on hysteroscopy. One patient had cervical polyp, three had cervical stenosis. The prevalence of the findings however differ slightly form a study done in the Saudi Arabia [1] where out of the 58 patients in whom hysteroscopy was performed, normal cavities were found in $3 \%$ of the patients, endometrial polyps in $39 \%$ of the patients, submucous fibroids in $13 \%$ of the patients, a distorted cavity in $1 \%$ of the patients patient, and thickened endometrium in $1 \%$.

Overall all pathologies in this study were detected by SIS apart from Mullerian duct abnormality. However, Ludwin et al. [24] showed perfect diagnostic accuracy $(100.0 \%)$ in general detection of uterine abnormalities with 3D-SIS and was identical to Hysteroscopy [24]. Hence SIS is a more accurate than 3D-TVS in visualizing the endometrial cavity and is a better alternative to hysteroscopy. In the United Kingdom, Vathanan and Armar found SIS to be superior to TVS for the diagnosis of submucous fibroids and endometrial polyps and therefore suitable for assessing intracavity pathology after TVS examination [25].

SIS is superior to TVS for the diagnosis of endometrial polyps and submucous fibroids therefore should be considered as an intermediate investigation after TVS to assess intracavity pathology and to confirm the diagnosis; hysteroscopy should be considered if there is a therapeutic intervention to be performed.

Concerns have however been raised about hysteroscopy being not only an expensive and invasive procedure, but unnecessary in $50 \%$ of the women who had normal findings, suggesting 3D SIS as an initial alternative approach in investigating women with AUB [5] [11]. In a meta-analysis, a total of 2228 procedures were reviewed that compared 3D SIS with hysteroscopy [26] [27]. The pooled sensitivity of 3D SIS for evaluating uterine cavity was 0.95 ( $95 \%$ confidence interval [CI], $0.93-0.97$ ), and the pooled specificity was 0.88 (95\% CI, $0.85-0.92$ ). This meta-analysis suggested that SIS was an accurate means of evaluating the endometrial cavity in pre- and postmenopausal women with AUB and this concurred with the findings of this study.

In view of the above, SIS is more accurate than TVS in evaluation of intracavitary abnormalities amongst pre- and post-menopausal women. Therefore, a primary approach of SIS to evaluate an endometrial lesion would be an effective and alternative method to hysteroscopy.

\section{Conclusion}

SIS has a comparable diagnostic accuracy to DH and was superior to TVS in de- 
tecting uterine pathologies in patients presenting with abnormal uterine bleeding. The use of SIS as an investigational technique should therefore be considered as it also has a high specificity and exceptional PPV and NPV. Therefore, in resource limited countries such as in Africa, SIS should be the first line of diagnosis of endometrial pathologies and diagnostic hysteroscopy should be reserved for patients with inconclusive SIS.

\section{Acknowledgements}

Special thanks to Prof. Rafique Parkar and Dr. Khwasa.

My sincere appreciation goes to my supervisors: Prof. Ojwang, Dr. Wanyoike and Dr. Obimbo for their patience, understanding, and guidance throughout this project. I thank them for their tireless efforts, supervision, encouragement, and for sacrificing long hours to ensure that my dissertation goes through.

I would like to express my sincere gratitude to Prof. Rafique Parkar, Dr. David Otieno, Dr. Giddie and Dr. Khwasa, for their remarkable support in assisting me with my procedures, ensuring I that I completed my research on time, Mr. Wycliffe Ayieko, who helped me with my data collection, and statistics and Dr. Christosm Barasa who diligently worked on my data and made it look as it does today. Special thanks to the clients who took part in the study and I hope through them there will be improved healthcare.

I would also like to acknowledge the Department of Obstetrics and Gynecology and my lecturers for their assistance and guidance that leads me to realize my dream.

May God bless you.

\section{Conflicts of Interest}

The authors declare no conflicts of interest regarding the publication of this paper.

\section{References}

[1] Khan, F., Jamaat, S. and Al-Jaroudi, D. (2011) Saline Infusion Sonohysterography versus Hysteroscopy for Uterine Cavity Evaluation. Annals of Saudi Medicine, 31, 387-392. https://doi.org/10.4103/0256-4947.83213

[2] Babacan, A., Gun, I., Kizilaslan, C., Ozden, O., Muhcu, M., Mungen, E., et al. (2014) Comparison of Transvaginal Ultrasonography and Hysteroscopy in the Diagnosis of Uterine Pathologies. International Journal of Clinical and Experimental Medicine, 7, 764-769.

[3] Kelekci, S., Kaya, E., Alan, M., Alan, Y., Bilge, U. and Mollamahmutoglu, L. (2005) Comparison of Transvaginal Sonography, Saline Infusion Sonography, and Office Hysteroscopy in Reproductive-Aged Women with or without Abnormal Uterine Bleeding. Fertility and Sterility, 84, 682-686. https://doi.org/10.1016/j.fertnstert.2005.03.036

[4] Rezk, M. and Shawky, M. (2015) The Safety and Acceptability of Saline Infusion Sonography versus Hysterosalpingography for Evaluation of Tubal Patency in Infertile Women. Middle East Fertility Society Journal, 20, 108-113. 
https://doi.org/10.1016/j.mefs.2014.06.003

[5] Seshadri, S., Khalil, M., Osman, A., Clough, A., Jayaprakasan, K. and Khalaf, Y. (2015) The Evolving Role of Saline Infusion Sonography (SIS) in Infertility. The European Journal of Obstetrics \& Gynecology and Reproductive Biology, 185, 66-73. https://doi.org/10.1016/j.ejogrb.2014.11.037

[6] Rudra, S., Duggal, B. and Bharadwaj, D. (2009) Prospective Study of Saline Infusion Sonography and Office Hysteroscopy. Medical Journal Armed Forces India, 65, 332-335. https://doi.org/10.1016/S0377-1237(09)80094-4

[7] Krampl, E., Bourne, T., Hurlen-Solbakken, H. and Istre, O. (2001) Transvaginal Ultrasonography Sonohysterography and Operative Hysteroscopy for the Evaluation of Abnormal Uterine Bleeding. Acta Obstetricia et Gynecologica Scandinavica, 80, 616-622. https://doi.org/10.1034/j.1600-0412.2001.800706.x

[8] Goyal, B.K., Gaur, I., Sharma, S., Saha, A. and Das, N.K. (2015) Transvaginal Sonography versus Hysteroscopy in Evaluation of Abnormal Uterine Bleeding. Medical Journal Armed Forces India, 71, 120-125.

https://doi.org/10.1016/j.mjafi.2014.12.001

[9] Pasrija, S., Trivedi, S.S. and Narula, M.K. (2004) Prospective Study of Saline Infusion Sonohysterography in Evaluation of Perimenopausal and Postmenopausal Women with Abnormal Uterine Bleeding. Journal of Obstetrics and Gynaecology Research, 30, 27-33. https://doi.org/10.1111/j.1341-8076.2004.00151.x

[10] Widrich, T., Bradley, L.D., Mitchinson, A.R. and Collins, R.L. (1996) Comparison of Saline Infusion Sonography with Office Hysteroscopy for the Evaluation of the Endometrium. American Journal of Obstetrics \& Gynecology, 174, 1327-1334. https://doi.org/10.1016/S0002-9378(96)70680-4

[11] Heliövaara-Peippo, S., Hurskainen, R., Teperi, J., Aalto, A.-M., Grénman, S., Halmesmäki, K., et al. (2014) Quality of Life and Costs of Levonorgestrel-Releasing Intrauterine System or Hysterectomy in the Treatment of Menorrhagia. Obstetrical \& Gynecological Survey, 69, 204-205. https://doi.org/10.1097/01.ogx.0000446908.04052.57

[12] Yildizhan, B., Yildizhan, R., Ozkesici, B. and Suer, N. (2008) Transvaginal Ultrasonography and Saline Infusion Sonohysterography for the Detection of Intra-Uterine Lesions in Pre- and Post-Menopausal Women with Abnormal Uterine Bleeding. Journal of International Medical Research, 36, 1205-1213. https://doi.org/10.1177/147323000803600606

[13] Arora, V. and Quinn, M.A. (2012) Endometrial Cancer. Best Practice \& Research: Clinical Obstetrics \& Gynaecology, 26, 311-324. https://doi.org/10.1016/j.bpobgyn.2011.12.007

[14] Chawla, I., Tripathi, S., Vohra, P. and Singh, P. (2014) To Evaluate the Accuracy of Saline Infusion Sonohysterography (SIS) for Evaluation of Uterine Cavity Abnormalities in Patients with Abnormal Uterine Bleeding. The Journal of Obstetrics and Gynecology of India, 64, 197-201. https://doi.org/10.1007/s13224-013-0501-4

[15] Shiva, M., Ahmadi, F., Arabipoor, A., Oromiehchi, M. and Chehrazi, M. (2018) Accuracy of Two-Dimensional Transvaginal Sonography and Office Hysteroscopy for Detection of Uterine Abnormalities in Patients with Repeated Implantation Failures or Recurrent Pregnancy Loss. International Journal of Fertility \& Sterility, 11, 287-292.

[16] Farquhar, C., Ekeroma, A., Furness, S. and Arroll, B. (2003) A Systematic Review of Transvaginal Ultrasonography, Sonohysterography and Hysteroscopy for the Investigation of Abnormal Uterine Bleeding in Premenopausal Women. Acta Obstetricia 
et Gynecologica Scandinavica, 82, 493-504.

https://doi.org/10.1034/j.1600-0412.2003.00191.x

[17] Cepni, I., Ocal, P., Erkan, S., Saricali, F.S., Akbas, H., Demirkiran, F., et al. (2005) Comparison of Transvaginal Sonography, Saline Infusion Sonography and Hysteroscopy in the Evaluation of Uterine Cavity Pathologies. The Australian and New Zealand Journal of Obstetrics and Gynaecology, 45, 30-35. https://doi.org/10.1111/j.1479-828X.2005.00336.x

[18] Dekroon, C., Debock, G., Dieben, S. and Jansen, F. (2003) Saline Contrast Hysterosonography in Abnormal Uterine Bleeding: A Systematic Review and Meta-Analysis. BJOG: An International Journal of Obstetrics and Gynaecology, 110, 938-947. https://doi.org/10.1016/S1470-0328(03)02472-8

[19] Radwan, P., Radwan, M., Kozarzewski, M., Polac, I. and Wilczyński, J. (2014) Evaluation of Sonohysterography in Detecting Endometrial Polyps-241 Cases Followed with Office Hysteroscopies Combined with Histopathological Examination. Videosurgery and Other Miniinvasive Techniques, 3, 344-350. https://doi.org/10.5114/wiitm.2014.43024

[20] Rani, P.R., Haritha, P.H. and Gowri, R. (2007) Comparative Study of Transperineal and Transabdominal Sonography in the Diagnosis of Placenta Previa. Journal of Obstetrics and Gynaecology Research, 33, 134-137. https://doi.org/10.1111/j.1447-0756.2007.00505.x

[21] Dijkhuizen, F. (1996) The Accuracy of Transvaginal Ultrasonography in the Diagnosis of Endometrial Abnormalities. Obstetrics \& Gynecology, 87, 345-349. https://doi.org/10.1016/0029-7844(95)00450-5

[22] Shaikh, F.S. (2017) Correlation of Ultrasonography, Hysterosalpingography and Hystero-Laparoscopy Findings in Cases of Infertility. International Journal of Reproduction, Contraception, Obstetrics and Gynecology, 6, 5390. https://doi.org/10.18203/2320-1770.ijrcog20175248

[23] Seshadri, S., El-Toukhy, T., Douiri, A., Jayaprakasan, K. and Khalaf, Y. (2015) Diagnostic Accuracy of Saline Infusion Sonography in the Evaluation of Uterine Cavity Abnormalities Prior to Assisted Reproductive Techniques: A Systematic Review and Meta-Analyses. Human Reproduction Update, 21, 262-274. https://doi.org/10.1093/humupd/dmu057

[24] Ludwin, A., Ludwin, I., Pity ski, K., Banas, T. and Jach, R. (2014) Role of Morphologic Characteristics of the Uterine Septum in the Prediction and Prevention of Abnormal Healing Outcomes after Hysteroscopic Metroplasty. Human Reproduction, 29, 1420-1431. https://doi.org/10.1093/humrep/deu110

[25] Vathanan, V. and Armar, N.A. (2016) A Comparative Observational Study of the Use of Saline Uterine Hydrosonography for the Diagnosis and Assessment of Uterine Cavity Lesions in Women. International Journal of Reproductive Medicine, 2016, Article ID: 9317194. https://doi.org/10.1155/2016/9317194

[26] de Kroon, C.D., de Bock, G.H., Dieben, S.W.M. and Jansen, F.W. (2004) Saline Contrast Hysterosonography in Abnormal Uterine Bleeding: A Systematic Review and Meta-Analysis. Obstetrical \& Gynecological Survey, 59, 265-266.

https://doi.org/10.1097/01.OGX.0000119181.63501.35

[27] Buderer, N.M. (1996) Statistical Methodology: I Incorporating the Prevalence of Disease into the Sample Size Calculation for Sensitivity and Specificity. Academic Emergency Medicine, 3, 895-900.

https://doi.org/10.1111/j.1553-2712.1996.tb03538.x 